\title{
Toward uncertain finance theory
}

\author{
Baoding Liu
}

Correspondence:

liu@tsinghua.edu.cn

Baoding Liu, Uncertainty Theory

Laboratory, Department of

Mathematical Sciences, Tsinghua

University, Beijing 100084, China

\begin{abstract}
This paper first introduces a paradox of stochastic finance theory that shows the real stock price is impossible to follow any Ito's stochastic differential equation. After a survey on uncertainty theory, uncertain process, uncertain calculus, and uncertain differential equation, this paper discusses some possible applications of uncertain differential equations to financial markets. Finally, it is suggested that a new uncertain finance theory should be developed based on uncertainty theory and uncertain differential equation.
\end{abstract}

Keywords: Finance, Uncertainty theory, Uncertain process, Uncertain calculus, Uncertain differential equation

\section{Review}

When no samples are available to estimate a probability distribution, we have to invite some domain experts to evaluate their belief degree that each event will occur. Perhaps some people think that personal belief degree is subjective probability or fuzzy concept. However, Liu [1] declared that it is inappropriate because both probability theory and fuzzy set theory may lead to counterintuitive results in this case. In order to rationally deal with the belief degree, an uncertainty theory was founded by Liu [2] and subsequently studied by many scholars. Nowadays, uncertainty theory has become a branch of axiomatic mathematics for modeling human uncertainty.

Based on uncertainty theory, the concept of uncertain process was given by Liu [3] as a sequence of uncertain variables indexed by time. Besides, the concept of uncertain integral was also proposed by Liu [3] in order to integrate an uncertain process with respect to a canonical process. Furthermore, Liu [4] recast his work via the fundamental theorem of uncertain calculus and thus produced the techniques of chain rule, change of variables, and integration by parts. Since then, the theory of uncertain calculus was well developed.

After uncertain differential equation was proposed by Liu [3] as a differential equation involving uncertain process, an existence and uniqueness theorem of a solution of uncertain differential equation was proved by Chen and Liu [5] under linear growth condition and Lipschitz continuous condition. The theorem was verified again by Gao [6] under local linear growth condition and local Lipschitz continuous condition. In order to solve uncertain differential equations, Chen and Liu [5] obtained an analytic solution to linear uncertain differential equations. In addition, Liu [7] presented a spectrum of analytic methods to solve some special classes of nonlinear uncertain differential equations. More importantly, Yao and Chen [8] showed that the solution of an uncertain differential

(C) 2013 Liu; licensee Springer. This is an Open Access article distributed under the terms of the Creative Commons Attribution License (http://creativecommons.org/licenses/by/2.0), which permits unrestricted use, distribution, and reproduction in any medium, provided the original work is properly cited. 
equation can be represented by a family of solutions of ordinary differential equations, thus relating uncertain differential equations and ordinary differential equations. On the basis of the Yao-Chen formula, a numerical method was also designed by Yao and Chen [8] for solving general uncertain differential equations. Furthermore, Yao [9] presented some formulas to calculate the extreme values, first hitting time and integral of solution of uncertain differential equation.

Uncertain differential equations were first introduced into finance by Liu [4] in which an uncertain stock model was proposed and European option price formulas were documented. Besides, Chen [10] derived American option price formulas for this type of uncertain stock model. In addition, Peng and Yao [11] presented a different uncertain stock model and obtained the corresponding option price formulas, and Yu [12] proposed an uncertain stock model with jumps. Uncertain differential equations were also employed to model uncertain currency markets by Liu and Chen [13] in which an uncertain currency model was proposed. Uncertain differential equations were used to simulate interest rate by Chen and Gao [14], and an uncertain interest rate model was presented. On the basis of this model, the price of zero-coupon bond was also produced. Uncertain differential equations were applied to optimal control by Zhu [15] in which Zhu's equation of optimality is proved to be a necessary condition for extremum of uncertain optimal control model.

This paper first introduces a paradox of stochastic finance theory. After a survey on uncertainty theory, uncertain process, uncertain calculus, and uncertain differential equation, this paper shows some possible applications of uncertain differential equations to financial markets. Finally, this paper suggests to develop an uncertain finance theory by using uncertainty theory and uncertain differential equation.

\section{A paradox of stochastic finance theory}

The origin of stochastic finance theory can be traced to Louis Bachelier's doctoral dissertation Théorie de la Speculation in 1900. However, Bachelier's work had little impact for more than a half century. After Kiyosi Ito invented stochastic calculus [16] and stochastic differential equation [17], stochastic finance theory was well developed among others by Samuelson [18], Black and Scholes [19], and Merton [20] during the 1960s and 1970s.

Traditionally, stochastic finance theory presumes that the stock price (including currency exchange rate and interest rate) follows an Ito's stochastic differential equation. Is it really reasonable? In fact, this widely accepted presumption was continuously challenged by many scholars. Let us assume that the stock price $X_{t}$ follows the stochastic differential equation

$$
\mathrm{d} X_{t}=e X_{t} \mathrm{~d} t+\sigma X_{t} \mathrm{~d} W_{t}
$$

where $e$ is the $\log$-drift, $\sigma$ is the log-diffusion, and $W_{t}$ is a Wiener process. Let us see what will happen with such an assumption. It follows from the stochastic differential equation (1) that $X_{t}$ is a geometric Wiener process, i.e.,

$$
X_{t}=X_{0} \exp \left(\left(e-\sigma^{2} / 2\right) t+\sigma W_{t}\right)
$$

from which we derive

$$
W_{t}=\frac{\ln X_{t}-\ln X_{0}-\left(e-\sigma^{2} / 2\right) t}{\sigma}
$$


whose increment is

$$
\Delta W_{t}=\frac{\ln X_{t+\Delta t}-\ln X_{t}-\left(e-\sigma^{2} / 2\right) \Delta t}{\sigma} .
$$

Write

$$
A=-\frac{\left(e-\sigma^{2} / 2\right) \Delta t}{\sigma} .
$$

Note that the real stock price $X_{t}$ is actually a step function of time with a finite number of jumps although it looks like a curve. During a fixed period, without loss of generality, we assume that $X_{t}$ is observed to have 100 jumps. Now we divide the period into 10,000 equal intervals. Then we may observe 10,000 samples of $X_{t}$. It follows from Equation 4 that $\Delta W_{t}$ has 10,000 samples that consist of 9,900 A's and 100 other numbers:

$$
\underbrace{A, A, \cdots, A}_{9,900}, \underbrace{B, C, \cdots, Z}_{100} \text {. }
$$

Nobody can believe that those 10,000 samples follow a normal probability distribution with expected value 0 and variance $\Delta t$. See Figure 1 . This fact is in contradiction with the property of Wiener process that the increment $\Delta W_{t}$ is a normal random variable with expected value 0 and variance $\Delta t$. Therefore, the stock price $X_{t}$ does not follow the stochastic differential equation.

Perhaps some people think that the stock price does behave like a geometric Wiener process (or Ornstein-Uhlenbeck process) in macroscopy although they recognize the paradox in microscopy. However, as the very core of stochastic finance theory, Ito's calculus is just built on the microscopic structure (i.e., the differential $\mathrm{d} W_{t}$ ) of Wiener process rather than macroscopic structure. More precisely, Ito's calculus is dependent on the presumption that $\mathrm{d} W_{t}$ is a normal random variable with expected value 0 and variance $\mathrm{d} t$. This unreasonable presumption is what causes the second order term in Ito's formula,

$$
\mathrm{d} X_{t}=\frac{\partial h}{\partial t}\left(t, W_{t}\right) \mathrm{d} t+\frac{\partial h}{\partial w}\left(t, W_{t}\right) \mathrm{d} W_{t}+\frac{1}{2} \frac{\partial^{2} h}{\partial w^{2}}\left(t, W_{t}\right) \mathrm{d} t .
$$

In fact, the increment of stock price is impossible to follow any continuous probability distribution. On the basis of the above paradox, personally, I do not think Ito's calculus can play the essential tool of finance theory because Ito's stochastic differential equation is impossible to model real stock price.

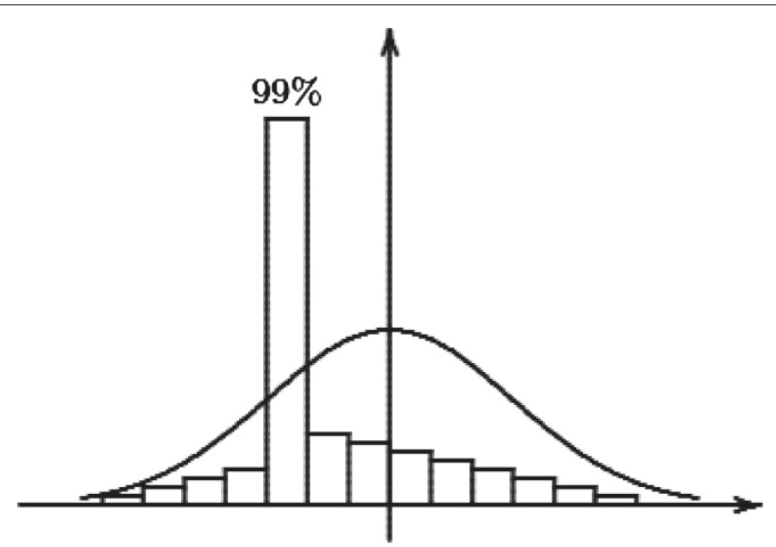

Figure 1 Normal distribution vs real frequency. 


\section{What is uncertainty theory?}

Let $\Gamma$ be a nonempty set, and $\mathcal{L}$ a $\sigma$-algebra over $\Gamma$. Each element $\Lambda$ in $\mathcal{L}$ is called an event. A set function $\mathcal{M}$ from $\mathcal{L}$ to $[0,1]$ is called an uncertain measure if it satisfies the following axioms [2]:

Axiom 1. (Normality axiom) $\mathcal{M}\{\Gamma\}=1$ for the universal set $\Gamma$;

Axiom 2. (Duality axiom) $\mathcal{N}\{\Lambda\}+\mathcal{N}\left\{\Lambda^{c}\right\}=1$ for any event $\Lambda$;

Axiom 3. (Subadditivity axiom) For every countable sequence of events $\Lambda_{1}, \Lambda_{2}, \cdots$, we have

$$
\mathcal{M}\left\{\bigcup_{i=1}^{\infty} \Lambda_{i}\right\} \leq \sum_{i=1}^{\infty} \mathcal{M}\left\{\Lambda_{i}\right\} .
$$

The triplet $(\Gamma, \mathcal{L}, \mathcal{M})$ is called an uncertainty space. In order to obtain an uncertain measure of compound event, a product uncertain measure was defined by Liu [4], thus producing the fourth axiom of uncertainty theory:

Axiom 4. (Product axiom) Let $\left(\Gamma_{k}, \mathcal{L}_{k}, \mathcal{M}_{k}\right)$ be uncertainty spaces for $k=1,2, \ldots$ The product uncertain measure $\mathcal{M}$ is an uncertain measure satisfying

$$
\mathcal{M}\left\{\prod_{k=1}^{\infty} \Lambda_{k}\right\}=\bigwedge_{k=1}^{\infty} \mathcal{M}_{k}\left\{\Lambda_{k}\right\}
$$

where $\Lambda_{k}$ are arbitrarily chosen events from $\mathcal{L}_{k}$ for $k=1,2, \ldots$, respectively.

An uncertain variable is defined by Liu [2] as a measurable function $\xi$ from an uncertainty space $(\Gamma, \mathcal{L}, \mathcal{M})$ to the set of real numbers, i.e., for any Borel set $B$ of real numbers, the set

$$
\{\xi \in B\}=\{\gamma \in \Gamma \mid \xi(\gamma) \in B\}
$$

is an event. In order to describe an uncertain variable in practice, the concept of uncertainty distribution is defined by Liu [2] as

$$
\Phi(x)=\mathcal{M}\{\xi \leq x\}, \quad \forall x \in \mathfrak{R} .
$$

Peng and Iwamura [21] proved that a function $\Phi: \mathfrak{R} \rightarrow[0,1]$ is an uncertainty distribution if and only if it is a monotone increasing function except $\Phi(x) \equiv 0$ and $\Phi(x) \equiv 1$.

An uncertainty distribution $\Phi(x)$ is said to be regular if it is a continuous and strictly increasing function with respect to $x$ at which $0<\Phi(x)<1$, and

$$
\lim _{x \rightarrow-\infty} \Phi(x)=0, \quad \lim _{x \rightarrow+\infty} \Phi(x)=1 .
$$

Let $\xi$ be an uncertain variable with regular uncertainty distribution $\Phi(x)$. Then the inverse function $\Phi^{-1}(\alpha)$ is called the inverse uncertainty distribution of $\xi$ [22].

It is easy to verify that $\Phi^{-1}(\alpha)$ is a continuous and strictly increasing function with respect to $\alpha \in(0,1)$. Conversely, suppose $\Phi^{-1}(\alpha)$ is a continuous and strictly increasing function on $(0,1)$. Define

$$
\Phi(x)=\left\{\begin{array}{l}
0, \text { if } x \leq \lim _{\alpha \downarrow 0} \Phi^{-1}(\alpha) \\
\alpha, \text { if } x=\Phi^{-1}(\alpha) \\
1, \text { if } x \geq \lim _{\alpha \uparrow 1} \Phi^{-1}(\alpha) .
\end{array}\right.
$$


It follows that $\Phi(x)$ is an uncertainty distribution of some uncertain variable $\xi$. Then for each $\alpha \in(0,1)$, we have

$$
\mathcal{M}\left\{\xi \leq \Phi^{-1}(\alpha)\right\}=\Phi\left(\Phi^{-1}(\alpha)\right)=\alpha .
$$

Thus, $\Phi^{-1}(\alpha)$ is just the inverse uncertainty distribution of the uncertain variable $\xi$. Hence, we have a sufficient and necessary condition of inverse uncertainty distribution: A function $\Phi^{-1}(\alpha):(0,1) \rightarrow \mathfrak{R}$ is an inverse uncertainty distribution if and only if it is a continuous and strictly increasing function with respect to $\alpha$.

The expected value of an uncertain variable $\xi$ is defined by Liu [2] as an average value of the uncertain variable in the sense of uncertain measure, i.e.,

$$
E[\xi]=\int_{0}^{+\infty} \mathcal{M}\{\xi \geq r\} \mathrm{d} r-\int_{-\infty}^{0} \mathcal{M}\{\xi \leq r\} \mathrm{d} r
$$

provided that at least one of the two integrals is finite. If $\xi$ has an uncertainty distribution $\Phi$, then the expected value may be calculated by

$$
E[\xi]=\int_{0}^{+\infty}(1-\Phi(x)) \mathrm{d} x-\int_{-\infty}^{0} \Phi(x) \mathrm{d} x .
$$

Independence is an extremely important concept in uncertainty theory. The uncertain variables $\xi_{1}, \xi_{2}, \cdots, \xi_{n}$ are said to be independent [4] if

$$
\mathcal{M}\left\{\bigcap_{i=1}^{n}\left(\xi_{i} \in B_{i}\right)\right\}=\bigwedge_{i=1}^{n} \mathcal{M}\left\{\xi_{i} \in B_{i}\right\}
$$

for any Borel sets $B_{1}, B_{2}, \cdots, B_{n}$ of real numbers. Equivalently, those uncertain variables are independent if and only if

$$
\mathcal{M}\left\{\bigcup_{i=1}^{n}\left(\xi_{i} \in B_{i}\right)\right\}=\bigvee_{i=1}^{n} \mathcal{M}\left\{\xi_{i} \in B_{i}\right\} .
$$

Let $\xi_{1}, \xi_{2}, \cdots, \xi_{n}$ be independent uncertain variables with uncertainty distributions $\Phi_{1}, \Phi_{2}, \cdots, \Phi_{n}$, respectively. If the function $f\left(x_{1}, x_{2}, \cdots, x_{n}\right)$ is strictly increasing with respect to $x_{1}, x_{2}, \cdots, x_{m}$ and strictly decreasing with respect to $x_{m+1}, x_{m+2}, \cdots, x_{n}$, then $\xi=f\left(\xi_{1}, \xi_{2}, \cdots, \xi_{n}\right)$ is an uncertain variable with inverse uncertainty distribution

$$
\Psi^{-1}(\alpha)=f\left(\Phi_{1}^{-1}(\alpha), \cdots, \Phi_{m}^{-1}(\alpha), \Phi_{m+1}^{-1}(1-\alpha), \cdots, \Phi_{n}^{-1}(1-\alpha)\right) .
$$

Then Liu and $\mathrm{Ha}[23]$ proved that the uncertain variable $\xi=f\left(\xi_{1}, \xi_{2}, \cdots, \xi_{n}\right)$ has an expected value

$$
E[\xi]=\int_{0}^{1} f\left(\Phi_{1}^{-1}(\alpha), \cdots, \Phi_{m}^{-1}(\alpha), \Phi_{m+1}^{-1}(1-\alpha), \cdots, \Phi_{n}^{-1}(1-\alpha)\right) \mathrm{d} \alpha .
$$

For exploring the details of uncertainty theory, the readers may consult Liu [24].

\section{Uncertain process}

Let $T$ be a totally ordered set (that is usually "time"), and let $(\Gamma, \mathcal{L}, \mathcal{M})$ be an uncertainty space. An uncertain process is defined by Liu [3] as a measurable function from $T \times(\Gamma, \mathcal{L}, \mathcal{M})$ to the set of real numbers, i.e., for each $t \in T$ and any Borel set $B$ of real numbers, the set

$$
\left\{X_{t} \in B\right\}=\left\{\gamma \in \Gamma \mid X_{t}(\gamma) \in B\right\}
$$

is an event. In other words, an uncertain process is a sequence of uncertain variables indexed by time. 
Note that if the index set $T$ becomes a partially ordered set (e.g., time $\times$ space, or a surface), then $X_{t}$ is called an uncertain field provided that $X_{t}$ is an uncertain variable at each point $t$. That is, an uncertain field is a generalization of an uncertain process.

An uncertain process $X_{t}$ is said to have an uncertainty distribution $\Phi_{t}(x)$ if at each time $t$, the uncertain variable $X_{t}$ has the uncertainty distribution $\Phi_{t}(x)$. It is easy to prove that $\Phi_{t}(x)$ is a monotone increasing function with respect to $x$ and $\Phi_{t}(x) \not \equiv 0, \Phi_{t}(x) \not \equiv 1$. Conversely, if at each time $t, \Phi_{t}(x)$ is a monotone increasing function except $\Phi_{t}(x) \equiv 0$ and $\Phi_{t}(x) \equiv 1$, it follows that there exists an uncertain variable $\xi_{t}$ whose uncertainty distribution is just $\Phi_{t}(x)$. Define

$$
X_{t}=\xi_{t}, \quad \forall t \in T .
$$

Then $X_{t}$ is an uncertain process and has the uncertainty distribution $\Phi_{t}(x)$. Thus, a function $\Phi_{t}(x): T \times \mathfrak{R} \rightarrow[0,1]$ is an uncertainty distribution of uncertain process if and only if at each time $t$, it is a monotone increasing function except $\Phi_{t}(x) \equiv 0$ and $\Phi_{t}(x) \equiv 1$.

An uncertainty distribution $\Phi_{t}(x)$ is said to be regular if at each time $t$, it is a continuous and strictly increasing function with respect to $x$ at which $0<\Phi_{t}(x)<1$, and

$$
\lim _{x \rightarrow-\infty} \Phi_{t}(x)=0, \quad \lim _{x \rightarrow+\infty} \Phi_{t}(x)=1 .
$$

Let $X_{t}$ be an uncertain process with regular uncertainty distribution $\Phi_{t}(x)$. Then the inverse function $\Phi_{t}^{-1}(\alpha)$ is called the inverse uncertainty distribution of $X_{t}$. It is easy to prove that $\Phi_{t}^{-1}(\alpha)$ is a continuous and strictly increasing function with respect to $\alpha \in(0,1)$. Conversely, if $\Phi_{t}^{-1}(\alpha)$ is a continuous and strictly increasing function with respect to $\alpha \in(0,1)$, it follows that there exists an uncertain variable $\xi_{t}$ whose inverse uncertainty distribution is just $\Phi_{t}^{-1}(\alpha)$. Define

$$
X_{t}=\xi_{t}, \quad \forall t \in T .
$$

Then $X_{t}$ is an uncertain process and has the inverse uncertainty distribution $\Phi_{t}^{-1}(\alpha)$. Hence, a function $\Phi_{t}^{-1}(\alpha): T \times(0,1) \rightarrow \mathfrak{R}$ is an inverse uncertainty distribution of uncertain process if and only if at each time $t$, it is a continuous and strictly increasing function with respect to $\alpha$.

An uncertain process $X_{t}$ is said to have independent increments if

$$
X_{t_{0}}, X_{t_{1}}-X_{t_{0}}, X_{t_{2}}-X_{t_{1}}, \cdots, X_{t_{k}}-X_{t_{k-1}}
$$

are independent uncertain variables where $t_{0}$ is the initial time and $t_{1}, t_{2}, \cdots, t_{k}$ are any times with $t_{0}<t_{1}<\cdots<t_{k}$. That is, an independent increment process means that its increments are independent uncertain variables whenever the time intervals do not overlap. Let $X_{t}$ be a sample-continuous independent increment process with an uncertainty distribution $\Phi_{t}(x)$ at each time $t$. When $f$ is a strictly increasing function, Liu [25] proved that the supremum

$$
\sup _{0 \leq t \leq s} f\left(X_{t}\right)
$$

has an uncertainty distribution

$$
\Psi(x)=\inf _{0 \leq t \leq s} \Phi_{t}\left(f^{-1}(x)\right) .
$$

This result is called the extreme value theorem of uncertain process. 
An uncertain process $X_{t}$ is said to have stationary increments if its increments are identically distributed uncertain variables whenever the time intervals have the same length, i.e., for any given $t>0$, the increments $X_{s+t}-X_{s}$ are identically distributed uncertain variables for all $s>0$.

Let $X_{t}$ be a stationary independent increment process with a crisp initial value $X_{0}$. Liu [22] showed that there exist two real numbers $a$ and $b$ such that the expected value

$$
E\left[X_{t}\right]=a+b t
$$

for any time $t \geq 0$. Furthermore, Chen [26] verified that there exists a real number $c$ such that the variance

$$
V\left[X_{t}\right]=c t^{2}
$$

for any time $t \geq 0$.

As an important type of uncertain process, a canonical process is a stationary independent increment process whose increments are normal uncertain variables. More precisely, an uncertain process $C_{t}$ is called a canonical process by Liu [4] if (1) $C_{0}=0$ and almost all sample paths are Lipschitz continuous, (2) $C_{t}$ has stationary and independent increments, and (3) every increment $C_{s+t}-C_{s}$ is a normal uncertain variable with expected value 0 and variance $t^{2}$.

It is easy to verify that the canonical process $C_{t}$ is a normal uncertain variable with expected value 0 and variance $t^{2}$ and has an uncertainty distribution

$$
\Phi(x)=\left(1+\exp \left(-\frac{\pi x}{\sqrt{3} t}\right)\right)^{-1}
$$

at each time $t>0$. In addition, for each time $t>0$, the ratio $C_{t} / t$ is a normal uncertain variable with expected value 0 and variance 1 . That is,

$$
\frac{C_{t}}{t} \sim \mathcal{N}(0,1)
$$

for any $t>0$.

What is the difference between canonical process and the Wiener process? First, canonical process is an uncertain process while the Wiener process is a stochastic process. Second, almost all sample paths of canonical process are Lipschitz continuous functions while almost all sample paths of the Wiener process are continuous but non-Lipschitz functions. Third, canonical process has a variance $t^{2}$ while the Wiener process has a variance $t$ at each time $t$.

\section{Uncertain calculus}

Uncertain calculus is a branch of mathematics that deals with differentiation and integration of uncertain processes. The key concept in uncertain calculus is the uncertain integral that allows us to integrate an uncertain process (the integrand) with respect to the canonical process (the integrator). The result of the uncertain integral is another uncertain process.

Let $X_{t}$ be an uncertain process and let $C_{t}$ be a canonical process. For any partition of closed interval $[a, b]$ with $a=t_{1}<t_{2}<\cdots<t_{k+1}=b$, the mesh is written as

$$
\Delta=\max _{1 \leq i \leq k}\left|t_{i+1}-t_{i}\right| .
$$


Then the uncertain integral of $X_{t}$ with respect to $C_{t}$ is defined by Liu [4] as

$$
\int_{a}^{b} X_{t} \mathrm{~d} C_{t}=\lim _{\Delta \rightarrow 0} \sum_{i=1}^{k} X_{t_{i}} \cdot\left(C_{t_{i+1}}-C_{t_{i}}\right)
$$

provided that the limit exists almost surely and is finite. Since $X_{t}$ and $C_{t}$ are uncertain variables at each time $t$, the limit in Equation 29 is also an uncertain variable.

Let $Z_{t}$ be an uncertain process. If there exist two uncertain processes $\mu_{t}$ and $\sigma_{t}$ such that

$$
Z_{t}=Z_{0}+\int_{0}^{t} \mu_{s} \mathrm{~d} s+\int_{0}^{t} \sigma_{s} \mathrm{~d} C_{s}
$$

for any $t \geq 0$, then we say $Z_{t}$ has an uncertain differential

$$
\mathrm{d} Z_{t}=\mu_{t} \mathrm{~d} t+\sigma_{t} \mathrm{~d} C_{t} .
$$

In this case, $Z_{t}$ is called an uncertain process with drift $\mu_{t}$ and diffusion $\sigma_{t}$. It is clear that uncertain integral and differential are mutually inverse operations. Please also note that an uncertain differential of an uncertain process has two parts, the " $\mathrm{d} t$ " part and the " $\mathrm{d} C_{t}$ " part.

Let $h(t, c)$ be a continuously differentiable function. Liu [4] showed that the uncertain process $Z_{t}=h\left(t, C_{t}\right)$ has an uncertain differential

$$
\mathrm{d} Z_{t}=\frac{\partial h}{\partial t}\left(t, C_{t}\right) \mathrm{d} t+\frac{\partial h}{\partial c}\left(t, C_{t}\right) \mathrm{d} C_{t} .
$$

This result is called the fundamental theorem of uncertain calculus.

Example 1. Let us calculate the uncertain differential of $t C_{t}$. In this case, we have $h(t, c)=$ $t c$ whose partial derivatives are

$$
\frac{\partial h}{\partial t}(t, c)=c, \quad \frac{\partial h}{\partial c}(t, c)=t .
$$

It follows from the fundamental theorem of uncertain calculus that

$$
\mathrm{d}\left(t C_{t}\right)=C_{t} \mathrm{~d} t+t \mathrm{~d} C_{t} .
$$

Example 2. Let us calculate the uncertain differential of $C_{t}^{2}$. In this case, we have $h(t, c)=$ $c^{2}$ whose partial derivatives are

$$
\frac{\partial h}{\partial t}(t, c)=0, \quad \frac{\partial h}{\partial c}(t, c)=2 c .
$$

It follows from the fundamental theorem of uncertain calculus that

$$
\mathrm{d} C_{t}^{2}=2 C_{t} \mathrm{~d} C_{t} .
$$

Example 3. Let $f(c)$ be a continuously differentiable function. Then we have

$$
\frac{\partial f}{\partial t}(c)=0, \quad \frac{\partial f}{\partial c}(c)=f^{\prime}(c) .
$$

It follows from the fundamental theorem of uncertain calculus that the uncertain process $f\left(C_{t}\right)$ has an uncertain differential

$$
\mathrm{d} f\left(C_{t}\right)=f^{\prime}\left(C_{t}\right) \mathrm{d} C_{t} .
$$

This formula is also called the chain rule of uncertain calculus.

As supplements to uncertain integral, Liu and Yao [27] suggested an uncertain integral with respect to multiple canonical processes. More generally, Chen and Ralescu [28] presented an uncertain integral with respect to the general Liu process. 


\section{Uncertain differential equation}

The study of uncertain differential equation was pioneered by Liu [3]. Nowadays, uncertain differential equation has achieved fruitful results in both theory and practice. Let $f$ and $g$ be two functions. Then

$$
\mathrm{d} X_{t}=f\left(t, X_{t}\right) \mathrm{d} t+g\left(t, X_{t}\right) \mathrm{d} C_{t}
$$

is called an uncertain differential equation. A solution is an uncertain process $X_{t}$ that satisfies Equation 36 identically in $t$.

Some analytic methods have been proposed for solving uncertain differential equations. For example, Chen and Liu [5] showed that the linear uncertain differential equation

$$
\mathrm{d} X_{t}=\left(u_{1 t} X_{t}+u_{2 t}\right) \mathrm{d} t+\left(v_{1 t} X_{t}+v_{2 t}\right) \mathrm{d} C_{t}
$$

has a solution

$$
X_{t}=U_{t}\left(X_{0}+\int_{0}^{t} \frac{u_{2 s}}{U_{s}} \mathrm{~d} s+\int_{0}^{t} \frac{v_{2 s}}{U_{s}} \mathrm{~d} C_{s}\right)
$$

where

$$
U_{t}=\exp \left(\int_{0}^{t} u_{1 s} \mathrm{~d} s+\int_{0}^{t} v_{1 s} \mathrm{~d} C_{s}\right) .
$$

In addition, Liu [7] verified that the nonlinear uncertain differential equation like

$$
\mathrm{d} X_{t}=f\left(t, X_{t}\right) \mathrm{d} t+\sigma_{t} X_{t} \mathrm{~d} C_{t}
$$

has a solution

$$
X_{t}=Y_{t}^{-1} Z_{t}
$$

where

$$
Y_{t}=\exp \left(-\int_{0}^{t} \sigma_{s} \mathrm{~d} C_{s}\right)
$$

and $Z_{t}$ is the solution of uncertain differential equation

$$
\mathrm{d} Z_{t}=Y_{t} f\left(t, Y_{t}^{-1} Z_{t}\right) \mathrm{d} t
$$

with initial value $Z_{0}=X_{0}$.

An important contribution to uncertain differential equation is the existence and uniqueness theorem by Chen and Liu [5]. An uncertain differential equation has a unique solution if the coefficients $f(t, x)$ and $g(t, x)$ satisfy linear growth condition

$$
|f(t, x)|+|g(t, x)| \leq L(1+|x|), \quad \forall x \in \Re, t \geq 0
$$

and Lipschitz condition

$$
|f(t, x)-f(t, y)|+|g(t, x)-g(t, y)| \leq L|x-y|, \quad \forall x, y \in \Re, t \geq 0
$$

for some constant L. Moreover, the solution is sample-continuous.

The concept of stability was given by Liu [4]. An uncertain differential equation is said to be stable if for any two solutions $X_{t}$ and $Y_{t}$, we have

$$
\lim _{\left|X_{0}-Y_{0}\right| \rightarrow 0} \mathcal{M}\left\{\left|X_{t}-Y_{t}\right|>\varepsilon\right\}=0, \quad \forall t>0
$$


for any given number $\varepsilon>0$. Yao et al. [29] proved that the uncertain differential equation is stable if the coefficients $f(t, x)$ and $g(t, x)$ satisfy linear growth condition

$$
|f(t, x)|+|g(t, x)| \leq K(1+|x|), \quad \forall x \in \mathfrak{R}, t \geq 0
$$

for some constant $K$ and strong Lipschitz condition

$$
|f(t, x)-f(t, y)|+|g(t, x)-g(t, y)| \leq L(t)|x-y|, \quad \forall x, y \in \Re, t \geq 0
$$

for some bounded and integrable function $L(t)$ on $[0,+\infty)$.

Uncertain differential equation has been extended by many scholars. For example, uncertain delay differential equation was studied among others by Barbacioru [30], Ge and Zhu [31], and Liu and Fei [32]. In addition, uncertain differential equation with jumps was suggested by Yao [33], and backward uncertain differential equation was discussed by Ge and Zhu [34].

\section{Numerical method}

Let $\alpha$ be a number with $0<\alpha<1$. An uncertain differential equation

$$
\mathrm{d} X_{t}=f\left(t, X_{t}\right) \mathrm{d} t+g\left(t, X_{t}\right) \mathrm{d} C_{t}
$$

is said to have an $\alpha$-path $X_{t}^{\alpha}$ if it solves the corresponding ordinary differential equation

$$
\mathrm{d} X_{t}^{\alpha}=f\left(t, X_{t}^{\alpha}\right) \mathrm{d} t+\left|g\left(t, X_{t}^{\alpha}\right)\right| \Phi^{-1}(\alpha) \mathrm{d} t
$$

where $\Phi^{-1}(\alpha)$ is the inverse uncertainty distribution of standard normal uncertain variable, i.e.,

$$
\Phi^{-1}(\alpha)=\frac{\sqrt{3}}{\pi} \ln \frac{\alpha}{1-\alpha} .
$$

Then

$$
\begin{aligned}
& \mathcal{M}\left\{X_{t} \leq X_{t}^{\alpha}, \forall t\right\}=\alpha, \\
& \mathcal{M}\left\{X_{t}>X_{t}^{\alpha}, \forall t\right\}=1-\alpha .
\end{aligned}
$$

This result is called the Yao-Chen formula [8]. In addition, at each time $t$, the solution $X_{t}$ has an inverse uncertainty distribution

$$
\Psi_{t}^{-1}(\alpha)=X_{t}^{\alpha} .
$$

Furthermore, for any monotone (increasing or decreasing) function $J$, we have

$$
E\left[J\left(X_{t}\right)\right]=\int_{0}^{1} J\left(X_{t}^{\alpha}\right) \mathrm{d} \alpha .
$$

The Yao-Chen formula relates uncertain differential equations and ordinary differential equations, just like that Feynman-Kac formula relates stochastic differential equations and partial differential equations.

It is almost impossible to find analytic solutions for general uncertain differential equations. This fact provides a motivation to design a numerical method to solve general uncertain differential equation

$$
\mathrm{d} X_{t}=f\left(t, X_{t}\right) \mathrm{d} t+g\left(t, X_{t}\right) \mathrm{d} C_{t} .
$$


In order to do so, a key point is to obtain an inverse uncertainty distribution $\Psi_{t}^{-1}(\alpha)$ of its solution $X_{t}$ at any given time $t$. For this purpose, Yao and Chen [8] designed the following algorithm:

Step 1. Fix $\alpha$ on $(0,1)$.

Step 2. Solve $\mathrm{d} X_{t}^{\alpha}=f\left(t, X_{t}^{\alpha}\right) \mathrm{d} t+\left|g\left(t, X_{t}^{\alpha}\right)\right| \Phi^{-1}(\alpha) \mathrm{d} t$ by any method of ordinary differential equation and obtain the $\alpha$-path $X_{t}^{\alpha}$, for example, by using the recursion formula

$$
X_{i+1}^{\alpha}=X_{i}^{\alpha}+f\left(t_{i}, X_{i}^{\alpha}\right) h+\left|g\left(t_{i}, X_{i}^{\alpha}\right)\right| \Phi^{-1}(\alpha) h
$$

where $\Phi$ is the standard normal uncertainty distribution and $h$ is the step length.

Step 3. The inverse uncertainty distribution of the solution $X_{t}$ is determined by

$$
\Psi_{t}^{-1}(\alpha)=X_{t}^{\alpha} .
$$

\section{Uncertain stock model}

Uncertain differential equations were first introduced into finance by Liu [4] in which an uncertain stock model was proposed,

$$
\left\{\begin{array}{l}
\mathrm{d} X_{t}=r X_{t} \mathrm{~d} t \\
\mathrm{~d} Y_{t}=e Y_{t} \mathrm{~d} t+\sigma Y_{t} \mathrm{~d} C_{t}
\end{array}\right.
$$

where $X_{t}$ is the bond price, $Y_{t}$ is the stock price, $r$ is the riskless interest rate, $e$ is the log-drift, $\sigma$ is the log-diffusion, and $C_{t}$ is a canonical process.

A European call option is a contract that gives the holder the right to buy a stock at an expiration time $s$ for a strike price $K$. The payoff from a European call option is $\left(Y_{s}-K\right)^{+}$ since the option is rationally exercised if and only if $Y_{s}>K$. Considering the time value of money resulted from the bond, the present value of this payoff is $\exp (-r s)\left(Y_{s}-K\right)^{+}$. Hence, the European call option price should be the expected present value of the payoff, i.e.,

$$
f_{c}=\exp (-r s) E\left[\left(Y_{s}-K\right)^{+}\right] .
$$

Liu [4] proved that

$$
f_{c}=\exp (-r s) Y_{0} \int_{K / Y_{0}}^{+\infty}\left(1+\exp \left(\frac{\pi(\ln y-e s)}{\sqrt{3} \sigma s}\right)\right)^{-1} \mathrm{~d} y .
$$

A European put option is a contract that gives the holder the right to sell a stock at an expiration time $s$ for a strike price $K$. The payoff from a European put option is $\left(K-Y_{s}\right)^{+}$ since the option is rationally exercised if and only if $Y_{s}<K$. Considering the time value of money resulted from the bond, the present value of this payoff is $\exp (-r s)\left(K-Y_{s}\right)^{+}$. Hence, the European put option price should be the expected present value of the payoff, i.e.,

$$
f_{p}=\exp (-r s) E\left[\left(K-Y_{s}\right)^{+}\right] .
$$

Liu [4] proved that

$$
f_{p}=\exp (-r s) Y_{0} \int_{0}^{K / Y_{0}}\left(1+\exp \left(\frac{\pi(e s-\ln y)}{\sqrt{3} \sigma s}\right)\right)^{-1} \mathrm{~d} y .
$$

An American call option is a contract that gives the holder the right to buy a stock at any time prior to an expiration time $s$ for a strike price $K$. It is clear that the payoff from an American call option is the supremum of $\left(Y_{t}-K\right)^{+}$over the time interval $[0, s]$. 
Considering the time value of money resulted from the bond, the present value of this payoff is

$$
\sup _{0 \leq t \leq s} \exp (-r t)\left(Y_{t}-K\right)^{+} .
$$

Hence, the American call option price should be the expected present value of the payoff, i.e.,

$$
f_{c}=E\left[\sup _{0 \leq t \leq s} \exp (-r t)\left(Y_{t}-K\right)^{+}\right] .
$$

Chen [10] proved that

$$
f_{c}=\exp (-r s) Y_{0} \int_{K / Y_{0}}^{+\infty}\left(1+\exp \left(\frac{\pi(\ln y-e s)}{\sqrt{3} \sigma s}\right)\right)^{-1} \mathrm{~d} y .
$$

An American put option is a contract that gives the holder the right to sell a stock at any time prior to an expiration time $s$ for a strike price $K$. It is clear that the payoff from an American put option is the supremum of $\left(K-Y_{t}\right)^{+}$over the time interval $[0, s]$. Considering the time value of money resulted from the bond, the present value of this payoff is

$$
\sup _{0 \leq t \leq s} \exp (-r t)\left(K-Y_{t}\right)^{+}
$$

Hence, the American put option price should be the expected present value of the payoff, i.e.,

$$
f_{p}=E\left[\sup _{0 \leq t \leq s} \exp (-r t)\left(K-Y_{t}\right)^{+}\right] .
$$

Chen [10] proved that

$$
f_{p}=\int_{0}^{K \exp (-r s)} \sup _{0 \leq t \leq s}\left(1+\exp \left(\frac{e}{\sqrt{3} \sigma}+\frac{\pi}{\sqrt{3} \sigma t} \ln \frac{Y_{0}}{K-y \exp (r t)}\right)\right)^{-1} \mathrm{~d} y .
$$

It is emphasized that other stock models were also actively investigated by Peng and Yao [11], $\mathrm{Yu}$ [12], and Chen et al. [35], among others.

\section{Uncertain currency model}

Liu and Chen [13] assumed that the exchange rate follows an uncertain differential equation and then proposed an uncertain currency model,

$$
\left\{\begin{array}{l}
\mathrm{d} X_{t}=u X_{t} \mathrm{~d} t \quad \text { (Domestic currency) } \\
\mathrm{d} Y_{t}=v Y_{t} \mathrm{~d} t \quad \text { (Foreign currency) } \\
\mathrm{d} Z_{t}=e Z_{t} \mathrm{~d} t+\sigma Z_{t} \mathrm{~d} C_{t} \quad \text { (Exchange rate) }
\end{array}\right.
$$

where $X_{t}$ represents the domestic currency with domestic interest rate $u, Y_{t}$ represents the foreign currency with foreign interest rate $v$, and $Z_{t}$ represents the exchange rate, that is, the domestic currency price of one unit of foreign currency at time $t$.

A currency option is a contract that gives the holder the right to exchange one unit of foreign currency at an expiration time $s$ for $K$ units of domestic currency. Suppose that the price of this contract is $f$ in domestic currency. Then the investor pays $f$ for buying 
the contract at time 0 and receives $\left(Z_{s}-K\right)^{+}$in domestic currency at the expiration time $s$. Thus, the expected return of the investor is

$$
-f+\exp (-u s) E\left[\left(Z_{s}-K\right)^{+}\right] .
$$

On the other hand, the bank receives $f$ for selling the contract at time 0 and pays $\left(1-K / Z_{s}\right)^{+}$in foreign currency at the expiration time $s$. Thus, the expected return of the bank is

$$
f-Z_{0} \exp (-v s) E\left[\left(1-K / Z_{s}\right)^{+}\right] .
$$

The fair price of this contract should make the investor and the bank have an identical expected return, i.e.,

$$
-f+\exp (-u s) E\left[\left(Z_{s}-K\right)^{+}\right]=f-Z_{0} \exp (-v s) E\left[\left(1-K / Z_{s}\right)^{+}\right] .
$$

Thus, the currency option price is

$$
f=\frac{1}{2} \exp (-u s) E\left[\left(Z_{s}-K\right)^{+}\right]+\frac{1}{2} \exp (-v s) Z_{0} E\left[\left(1-K / Z_{s}\right)^{+}\right] .
$$

Liu and Chen [13] proved that

$$
\begin{aligned}
f= & \frac{1}{2} \exp (-u s) Z_{0} \int_{K / Z_{0}}^{+\infty}\left(1+\exp \left(\frac{\pi(\ln y-e s)}{\sqrt{3} \sigma s}\right)\right)^{-1} \mathrm{~d} y \\
& +\frac{1}{2} \exp (-v s) Z_{0} \int_{0}^{1}\left(1+\exp \left(\frac{\pi\left(\ln \left(K / Z_{0}\right)-\ln y-e s\right)}{\sqrt{3} \sigma s}\right)\right)^{-1} \mathrm{~d} y .
\end{aligned}
$$

\section{Uncertain interest rate model}

Real interest rates do not remain unchanged. Chen and Gao [14] assumed that the interest rate $X_{t}$ follows an uncertain differential equation,

$$
\mathrm{d} X_{t}=\left(m-a X_{t}\right) \mathrm{d} t+\sigma \mathrm{d} C_{t}
$$

where $m, a$, and $\sigma$ are positive numbers, and $C_{t}$ is a canonical process.

A zero-coupon bond is a bond bought at a price lower than its face value, that is, the amount it promises to pay at the maturity date. For simplicity, we assume that the face value is always US\$1. Then the price of a zero-coupon bond with a maturity date $s$ is

$$
f=E\left[\exp \left(-\int_{0}^{s} X_{t} \mathrm{~d} t\right)\right] .
$$

Chen and Gao [14] proved that

$$
f=\frac{\sqrt{3} \sigma}{a}(s-g) \exp \left(-\frac{m s}{2 a}-\left(r_{0}-\frac{m}{a}\right) g\right) \csc \left(\frac{\sqrt{3} \sigma}{a}(s-g)\right)
$$

where

$$
g=\frac{1}{a}(1-\exp (-a s)) .
$$

\section{Uncertain finance theory}

At the beginning of this paper, a paradox was proposed to show that the real stock price is impossible to follow an Ito's stochastic differential equation. It follows from Figure 1 that the increments behave like an uncertain variable rather than a random variable. This fact motives us to model stock prices by uncertain differential equations. Personally, I think uncertain calculus may play a potential mathematical foundation of finance theory. 
If we say that the classical finance theory is a methodology dealing with financial markets by using probability theory, then uncertain finance theory is a methodology dealing with financial markets by using uncertainty theory. In addition to the uncertain stock models shown above, we may also accept other uncertain differential equations, for example,

$$
\begin{aligned}
& \mathrm{d} X_{t}=\left(m-a X_{t}\right) \mathrm{d} t+\sigma X_{t} \mathrm{~d} C_{t}, \\
& \mathrm{~d} X_{t}=\left(m-a X_{t}\right) \mathrm{d} t+\sigma \sqrt{X_{t}} \mathrm{~d} C_{t}, \\
& \mathrm{~d} X_{t}=\left(m-a X_{t}\right) \mathrm{d} t+\sigma \sqrt{b+X_{t}} \mathrm{~d} C_{t} .
\end{aligned}
$$

\section{Conclusions}

At first, a paradox of stochastic finance theory was introduced in this paper. After a survey on uncertainty theory, uncertain process, uncertain calculus, and uncertain differential equation, this paper summarized uncertain stock model, uncertain currency model, and uncertain interest model by using the tool of uncertain differential equation. Finally, it was suggested that an uncertain finance theory should be developed based on uncertainty theory.

\section{Competing interests}

The author declares that he has no competing interests.

\section{Acknowledgements}

This work was supported by the National Natural Science Foundation of China, grant no. 61273044

Received: 12 February 2013 Accepted: 18 February 2013

Published: 24 April 2013

\section{References}

1. Liu, B: Why is there a need for uncertainty theory? J. Uncertain Syst. 6(1), 3-10 (2012)

2. Liu, B: Uncertainty Theory. 2nd edn. Springer, Berlin (2007)

Liu, B: Fuzzy process, hybrid process and uncertain process. J. Uncertain Syst. 2(1), 3-16 (2008)

4. Liu, B: Some research problems in uncertainty theory. J. Uncertain Syst. 3(1), 3-10 (2009)

5. Chen, XW, Liu, B: Existence and uniqueness theorem for uncertain differential equations. Fuzzy Optimization Decis. Mak. 9(1), 69-81 (2010)

6. Gao, Y: Existence and uniqueness theorem on uncertain differential equations with local Lipschitz condition. J. Uncertain Syst. 6(3), 223-232 (2012)

7. Liu, YH: An analytic method for solving uncertain differential equations. J. Uncertain Syst. 6(4), 244-249 (2012)

8. Yao, K, Chen, XW: A numerical method for solving uncertain differential equations. J. Intell. Fuzzy Syst. (2013, in press)

9. Yao, K: Extreme values and integral of solution of uncertain differential equation. J. Uncertainty Anal. Appl. 1, Article $3(2013)$

10. Chen, XW: American option pricing formula for uncertain financial market. Int. J. Oper. Res. 8(2), 32-37 (2011)

11. Peng, J, Yao, K: A new option pricing model for stocks in uncertainty markets. Int J. Oper. Res. 8(2), 18-26 (2011)

12. Yu, XC: A stock model with jumps for uncertain markets. Int. J. Uncertainty Fuzziness Knowledge-Based Syst. 20(3), 421-432 (2012)

13. Liu, YH, Chen, XW: Uncertain currency model and currency option pricing. http://orsc.edu.cn/online/091010.pdf (2013)

14. Chen, XW, Gao, J: Uncertain term structure model of interest rate. Soft Comput. 17(4), 597-604 (2013)

15. Zhu, Y: Uncertain optimal control with application to a portfolio selection model. Cybern. Syst. 41(7), 535-547 (2010)

16. Ito, K: Stochastic integral. Proc. Jpn. Acad. Ser. A. 20(8), 519-524 (1944)

17. Ito, K: On stochastic differential equations. Mem. Am. Math. Soc. 4, 1-51 (1951)

18. Samuelson, PA: Rational theory of warrant pricing. Ind. Manage. Rev. 6, 13-31 (1965)

19. Black, F, Scholes, M: The pricing of option and corporate liabilities. J. Pol. Econ. 81, 637-654 (1973)

20. Merton, RC: Theory of rational option pricing. Bell J. Econ. Manage. Sci. 4, 141-183 (1973)

21. Peng, ZX, Iwamura, K: A sufficient and necessary condition of uncertainty distribution. J. Interdiscip. Math. 13(3), 277-285 (2010)

22. Liu, B: Uncertainty Theory: A Branch of Mathematics for Modeling Human Uncertainty. Springer, Berlin (2010)

23. Liu, YH, Ha, MH: Expected value of function of uncertain variables. J. Uncertain Syst. 4(3), 181-186 (2010)

24. Liu, B: Uncertainty Theory. 4th edn. http://orsc.edu.cn/liu/ut.pdf (2013) 
25. Liu, B: Extreme value theorems of uncertain process with application to insurance risk model. Soft Comput. 17(4), 549-556 (2013)

26. Chen, XW: Variation analysis of uncertain stationary independent increment process. Eur. J. Oper. Res. 222(2), 312-316 (2012)

27. Liu, B, Yao, K: Uncertain integral with respect to multiple canonical processes. J. Uncertain Syst. 6(4), 250-255 (2012)

28. Chen, XW, Ralescu, DA: Liu process and uncertain calculus. J. Uncertainty Anal. Appl. 1, Article 2 (2013)

29. Yao, K, Gao, J, Gao, Y: Some stability theorems of uncertain differential equation. Fuzzy Optimization Decis. Mak. 12(1), 3-13 (2013)

30. Barbacioru, IC: Uncertainty functional differential equations for finance. Surv Math. Appl. 5, 275-284 (2010)

31. Ge, XT, Zhu, Y: Existence and uniqueness theorem for uncertain delay differential equations. J. Comput. Inf. Syst. 8(20), 8341-8347 (2012)

32. Liu, HJ, Fei, WY: Neutral uncertain delay differential equations. Inf. Int. Interdiscip. J. 16(2), 1225-1232 (2013)

33. Yao, K: Uncertain calculus with renewal process. Fuzzy Optimization Decis. Mak. 11(3), 285-297 (2012)

34. Ge, XT, Zhu, Y: A necessary condition of optimality for uncertain optimal control problem. Fuzzy Optimization Decis. Mak. 12 (2013)

35. Chen, XW, Liu, YH, Ralescu, DA: Uncertain stock model with periodic dividends. Fuzzy Optimization Decis. Mak. 12(1), 111-123 (2013)

doi:10.1186/2195-5468-1-1

Cite this article as: Liu: Toward uncertain finance theory. Journal of Uncertainty Analysis Applications 2013 1:1.

\section{Submit your manuscript to a SpringerOpen ${ }^{\circ}$ journal and benefit from:}

$\checkmark$ Convenient online submission

$\checkmark$ Rigorous peer review

- Immediate publication on acceptance

- Open access: articles freely available online

- High visibility within the field

Retaining the copyright to your article

Submit your next manuscript at $\triangleright$ springeropen.com 